\title{
OPTIMAL CONTROL OF TEMPERATURE MODES OF THE INSTRUMENTAL CONSTRUCTIONS OF AUTONOMOUS OBJECTS
}

\author{
Mikhail Yu. Livshits ${ }^{1,}$, Boris.B. Borodulin ${ }^{1}$ \\ ${ }^{1}$ Samara State Technical University, Heat and Power Engineering Department, Russia
}

\begin{abstract}
Problem of the automatic thermogradient stabilization of the constructive elements sizes is discussed. Dimensional stability of instrumental constructions improves the validity of the indicated values of instruments located on these constructions. The optimal control problem is formulated. The optimal algorithm is proposed for variation of the intensity of the distributed controlled heat sources.
\end{abstract}

\section{Introduction}

Actual problem of the stabilization of temperature modes of load-bearing constructions of autonomous objects, on which the heat generating instruments of data measuring systems are located, was formulated and proved in [1]. Dimensional stability of the supporting constructions improves the validity of the indicated values of instruments located on these constructions; but thermal deformation due to heat generation of operating devices and the influence of external heat fluxes significantly increase the error of the measurements.

\section{Solution}

As it was found in [1], the measurement errors reduction can be achieved by implementation of a system for automatic thermogradient stabilization. The structural mathematical model of the controlled object is developed using the method of Green's functions [1-3].

Let us consider the linear formulation of the problem for the constructive element of a rectangular prism form with the sizes $x_{\max }, y_{\max }, z_{\max }$ for an isotropic material. Temperature field $\Theta(\rho, \varphi)$ at any point $\rho\left(l_{x}, l_{y}, l_{z}\right) \in \Omega_{\rho} \subset E^{3}$ of this constructive element can be described by inhomogeneous differential equation of heat transfer in a standard form [2,3]:

\footnotetext{
${ }^{\text {a }}$ Corresponding author: entcom@samgtu.ru
} 


$$
\frac{\Theta(\rho, \varphi)}{\partial \varphi}-\frac{\partial^{2} \Theta(\rho, \varphi)}{\partial l_{x}{ }^{2}}+\frac{\partial^{2} \Theta(\rho, \varphi)}{\partial l_{y}{ }^{2}}+\frac{\partial^{2} \Theta(\rho, \varphi)}{\partial l_{z}{ }^{2}}=\omega(\rho, \varphi)
$$

$\rho \in \Omega_{\rho}=\left\{l_{x}: l_{x} \in(0,1), l_{y}: l_{y} \in\left(0, l_{y_{\max }}\right), l_{z}: l_{z} \in\left(0, l_{z_{\max }}\right) ; \varphi \in(0, \infty) ;\right.$

with homogeneous boundary conditions:

$$
\begin{gathered}
\Theta\left(l_{x}, l_{y}, l_{z}, 0\right)=0, \varphi=0, \rho \in \bar{\Omega}_{\rho} ; \\
\frac{\partial \Theta\left(l_{x}, l_{y}, l_{z}, \varphi\right)}{\partial l_{x}}=\frac{\partial \Theta\left(l_{x}, l_{y}, l_{z}, \varphi\right)}{\partial l_{y}}=\frac{\partial \Theta\left(l_{x}, l_{y}, l_{z}, \varphi\right)}{\partial l_{z}}=0, \\
\varphi \in(0, \infty), \rho \in \partial \Omega_{\rho}, \partial \Omega_{\rho} \cup \Omega_{\rho}=\bar{\Omega}_{\rho} ;
\end{gathered}
$$

where the relative units are defined as follows: $l_{x}=x / x_{\max } ; l_{y}=y / x_{\max } ; l_{z}=z / x_{\max }$; $l_{y_{\text {max }}}=y_{\text {max }} / x_{\text {max }} ; l_{z_{\max }}=z_{\text {max }} / x_{\text {max }} ; \Theta\left(l_{x}, l_{y}, l_{z}, \varphi\right)=\lambda\left[\mathrm{T}\left(1_{\mathrm{x}}, 1_{\mathrm{y}}, 1_{\mathrm{z}}, \varphi\right)-\mathrm{T}_{\mathrm{dest}}\right] / \mathrm{x}^{2}{ }_{\text {max }} \mathrm{P}_{\text {max }} ;$ $\varphi=\lambda \tau / C x_{\text {max }}^{2}$. Here: $T\left(l_{x}, l_{y}, l_{z}, \varphi\right)$ - temperature of the constructive element, $T_{\text {dest }}$ - some basis value of temperature, for example, a required constant temperature, $\lambda$ - coefficient of thermal conductivity, $C$ - specific heat capacity, $P_{\max }$ - maximum volume density of heat sources. When the heat generating device is located on the surface of the construction, the standardizing function allows to reduce the initial inhomogeneous boundary problem to the standard form of (1) - (3) that can be written as follows with the help of application of the Green's function $[2,3]$ :

$$
\begin{aligned}
& \omega\left(l_{x}, l_{y}, l_{z}, \varphi\right)=\delta(\varphi) v\left(l_{x}, l_{y}, l_{z}\right)+\delta\left(l_{z}\right)\left\{\sum _ { j = 1 } ^ { N _ { 1 } } \mathrm { q } _ { j } ^ { ( 1 ) } ( \varphi ) \left[1 ( l _ { x } - a _ { 1 j } ) 1 ( - l _ { x } + b _ { 1 j } ) 1 \left(l_{y}-\right.\right.\right. \\
& \left.\left.\left.-c_{1 j}\right) 1\left(-l_{y}+d_{1 j}\right)\right]+\mathrm{q}^{(1)}\left(\varphi, \Theta_{\Pi_{1}}\right)+\mathrm{q}_{z}^{(1)}\left(\varphi, l_{x}, l_{y}\right)\right\}+\delta\left(l_{z}-l_{z_{\max }}\right)\left\{\sum _ { j = 1 } ^ { N _ { 2 } } \mathrm { q } _ { j } ^ { ( 2 ) } ( \varphi ) \left[1 \left(l_{x}-\right.\right.\right. \\
& \left.\left.\left.-a_{2 j}\right) 1\left(-l_{x}+b_{2 j}\right) 1\left(l_{y}-c_{2 j}\right) 1\left(-l_{y}+d_{2 j}\right)\right]+\mathrm{q}^{(2)}\left(\varphi, \Theta_{\Pi_{2}}\right)+\mathrm{q}_{z}^{(2)}\left(\varphi, l_{x}, l_{y}\right)\right\}+ \\
& \delta\left(l_{x}\right)\left\{\sum_{j=1}^{N_{3}} \mathrm{q}_{\mathrm{j}}^{(3)}(\varphi)\left[1\left(1_{\mathrm{y}}-\mathrm{c}_{3 \mathrm{j}}\right) 1\left(-\mathrm{l}_{\mathrm{y}}+\mathrm{d}_{3 \mathrm{j}}\right) 1\left(\mathrm{l}_{\mathrm{z}}-\mathrm{k}_{3 \mathrm{j}}\right) 1\left(-\mathrm{l}_{\mathrm{z}}+\mathrm{m}_{3 \mathrm{j}}\right)\right]+\mathrm{q}^{(3)}\left(\varphi, \Theta_{\Pi_{3}}\right)+\right. \\
& \left.\mathrm{q}_{\mathrm{x}}^{(3)}\left(\varphi, 1_{\mathrm{y}}, 1_{\mathrm{z}}\right)\right\}+\delta\left(1_{\mathrm{x}}-1\right)\left\{\sum _ { j = 1 } ^ { N _ { 4 } } \mathrm { q } _ { \mathrm { j } } ^ { ( 4 ) } ( \varphi ) \left[1 ( 1 _ { \mathrm { y } } - \mathrm { c } _ { 4 \mathrm { j } } ) 1 ( - \mathrm { l } _ { \mathrm { y } } + \mathrm { d } _ { 4 \mathrm { j } } ) 1 ( \mathrm { l } _ { \mathrm { z } } - \mathrm { k } _ { 4 \mathrm { j } } ) 1 \left(-\mathrm{l}_{\mathrm{z}}+\right.\right.\right. \\
& \left.\left.\left.+\mathrm{m}_{4 \mathrm{j}}\right)\right]+\mathrm{q}^{(4)}\left(\varphi, \Theta_{\Pi_{4}}\right)+\mathrm{q}_{x}^{(4)}\left(\varphi, l_{y}, l_{z}\right)\right\}+\delta\left(l_{y}\right)\left\{\sum _ { j = 1 } ^ { N _ { 5 } } \mathrm { q } _ { \mathrm { j } } ^ { ( 5 ) } ( \varphi ) \left[1 ( l _ { x } - a _ { 5 j } ) 1 \left(-l_{x}+\right.\right.\right. \\
& \left.\left.\left.+b_{5 j}\right) 1\left(l_{z}-k_{5 j}\right) 1\left(-l_{z}+m_{5 j}\right)\right]+\mathrm{q}^{(5)}\left(\varphi, \Theta_{\Pi_{5}}\right)+\mathrm{q}_{y}^{(5)}\left(\varphi, l_{x}, l_{z}\right)\right\}+\delta\left(l_{y}-\right. \\
& \left.-l_{y_{\max }}\right)\left\{\sum_{j=1}^{N_{6}} \mathrm{q}_{j}^{(6)}(\varphi)\left[1\left(l_{x}-a_{6 j}\right) 1\left(-l_{x}+b_{6 j}\right) 1\left(l_{z}-k_{6 j}\right) 1\left(-l_{z}++m_{6 j}\right)\right]+\right. \\
& \left.+\mathrm{q}^{(6)}\left(\varphi, \Theta_{\Pi_{6}}\right)+\mathrm{q}_{y}^{(6)}\left(\varphi, l_{x}, l_{z}\right)\right\}
\end{aligned}
$$


where $q^{(\alpha)}\left(\varphi, \Theta_{\Pi_{\alpha}}\right)$ - flow of heat exchange between the surface of $\alpha$-th side of the prism $(\alpha=1,6)$ and the corresponding surface of the environment with the temperature $\Theta_{\Pi_{\alpha}} ; \mathrm{q}_{\mathrm{j}}^{(\alpha)}(\varphi)$ - heat flow of $j$-th heat generating data measuring device from the set of all $\mathrm{N}_{\alpha}$ devices located on a rectangular surface, bounded by closed intervals $a_{\alpha j}, b_{\alpha j}, \alpha=1,2,5,6 ; c_{\alpha j}, d_{\alpha j}, \alpha=1,2,3,4 ; \mathrm{k}_{\alpha \mathrm{j}}, \mathrm{m}_{\alpha \mathrm{j}}$, $\alpha=3,4,5,6$; on $\alpha$-th verge; $q_{x}^{(\alpha)}\left(\varphi, l_{y}, l_{z}\right), q_{y}^{(\alpha)}\left(\varphi, l_{x}, l_{z}\right), q_{z}^{(\alpha)}\left(\varphi, l_{x}, l_{y}\right)$-distributed within $\alpha$-th verge heat flux $(\alpha=1,6)$ of the controlled heat sources of thermogradient stabilization system; $1(*)-$ Heaviside function (unit step function), $\delta(*)$ - delta function. According to the authors opinion, the most effective way is the formulation of optimal control problem for optimization of temperature field $\Theta\left(l_{x}, l_{y}, l_{z}, \varphi\right)$ of supporting constructions regarding typical optimization criterion.

Let us consider the possible ways of development of an automatic stabilization system. The dependence of thermal deformation of the structure on the temperature can be described by the known linear relations [5]. This fact does not allow using a quadratic deviation, $\left\|\Theta\left(l_{x}, l_{y}, l_{z}, \varphi_{k}\right)\right\|_{L_{2}\left[\rho \in \bar{\Omega}_{\rho}\right]}$, $\varphi_{k}=\lambda \tau_{k} / C x^{2}{ }_{\max }$ depending on $\mathrm{T}_{\text {dest }}$ to estimate the control quality of management of the resulting temperature field because this criterion is not sensitive to local thermal gradient, and thus to thermal deformation. Therefore, the Chebyshev norm is applied as optimization criterion in the optimal control problem:

$$
J=\left\|\Theta\left(l_{x}, l_{y}, l_{z}, \varphi_{k}\right)\right\|_{L_{\infty}\left[\rho \in \bar{\Omega}_{\rho}\right]}, \Omega_{\rho}=\left\{l_{x}: l_{x} \in[0,1], l_{y}: l_{y} \in\left[0, l_{y_{\max }}\right], l_{z}: l_{z} \in\left[0, l_{z_{\max }}\right]\right.
$$

Thus, we can formulate the optimal control problem as follows. It is required to determine the optimal law of variation of the heat flows $q_{x}^{(\alpha)}\left(\varphi, l_{y}, l_{z}\right), q_{y}^{(\alpha)}\left(\varphi, l_{x}, l_{z}\right), q_{z}^{(\alpha)}\left(\varphi, l_{x}, l_{y}\right)$ under the constraints on the permissible temperature $\Theta_{d}=$ const :

$$
\Theta(\rho, \varphi) \leq \Theta_{d}, \varphi \in(0, \infty), \rho \in \bar{\Omega}_{\rho}
$$

and on control resource:

$$
\begin{gathered}
\left|q_{\mathrm{x}}^{(\alpha)}\left(\varphi, 1_{\mathrm{y}}, 1_{\mathrm{z}}\right)\right| \leq 1 ; \alpha=3,4 ; 1_{\mathrm{x}}=0 ; 1_{\mathrm{x}}=1 ;\left|q_{\mathrm{y}}^{(\alpha)}\left(\varphi, 1_{\mathrm{x}}, 1_{\mathrm{z}}\right)\right| \leq 1 ; \alpha=5,6 ; 1_{\mathrm{y}}=0 ; \\
1_{\mathrm{y}}=1_{\mathrm{y}_{\max }} ;\left|q_{\mathrm{z}}^{(\alpha)}\left(\varphi, 1_{\mathrm{x}}, 1_{\mathrm{y}}\right)\right| \leq 1 ; \alpha=1,2 ; 1_{\mathrm{z}}=0 ; 1_{\mathrm{z}}=1_{\mathrm{z}_{\max }}
\end{gathered}
$$

when perturbations $q^{(\alpha)}\left(\varphi, \Theta_{\Pi_{\alpha}}\right), q_{j}^{(\alpha)}(\varphi), v\left(l_{x}, l_{y}, l_{z}\right)$ influence a control object (1) - (4). The searching optimal law of variation of the heat flows $q_{x}^{(\alpha)}\left(\varphi, l_{y}, l_{z}\right), q_{y}^{(\alpha)}\left(\varphi, l_{x}, l_{z}\right), q_{z}^{(\alpha)}\left(\varphi, l_{x}, l_{y}\right)$ should provide meeting the following conditions for an object (1)-(4) $J^{o p t}\left(q_{x(o p t)}^{(\alpha)}, q_{y(o p t)}^{(\alpha)}, q_{z(o p t)}^{(\alpha)}\right)=\min _{q_{x}^{(\alpha)}, q_{y}^{(\alpha)}, q_{z}^{(\alpha)}} J$ for a given value $\varphi=\varphi_{k}$ (problem of maximum accuracy $(\beta=1))$ or $\min _{q_{x}^{(\alpha)}, q_{y}^{(\alpha)}, q_{z}^{(\alpha)}} \varphi_{k}$ for a given value $J=J_{d o p}$ (time-optimal problem $(\beta=2)$ ). If the admissible control functions in (4) can be represented in the following multiplicative forms 


$$
\begin{gathered}
\mathrm{q}_{\mathrm{x}}^{(\alpha)}\left(\varphi, 1_{\mathrm{y}}, 1_{\mathrm{z}}\right)=\mathrm{U}_{\mathrm{x}}^{(\alpha)}(\varphi) \mathrm{V}_{\mathrm{x}}^{(\alpha)}\left(1_{\mathrm{y}}, 1_{\mathrm{z}}\right) ; \alpha=3,4 ; \mathrm{q}_{\mathrm{y}}^{(\alpha)}\left(\varphi, 1_{\mathrm{x}}, 1_{\mathrm{z}}\right)=\mathrm{U}_{\mathrm{y}}^{(\alpha)}(\varphi) \mathrm{V}_{\mathrm{y}}^{(\alpha)}\left(1_{\mathrm{x}}, 1_{\mathrm{z}}\right) ; \\
\alpha=5,6 ; \mathrm{q}_{\mathrm{z}}^{(\alpha)}\left(\varphi, 1_{\mathrm{x}}, 1_{\mathrm{y}}\right)=\mathrm{U}_{\mathrm{z}}^{(\alpha)}(\varphi) \mathrm{V}_{\mathrm{z}}^{(\alpha)}\left(1_{\mathrm{x}}, 1_{\mathrm{y}}\right) ; \alpha=1,2 ;
\end{gathered}
$$

with a priori prescribed functions $V_{x}^{(\alpha)}\left(l_{y}, l_{z}\right), V_{y}^{(\alpha)}\left(l_{x}, l_{z}\right), V_{z}^{(\alpha)}\left(l_{x}, l_{y}\right)$ the distribution of heat sources and if under these conditions it is possible in to write an optimal algorithms for variation of heat sources intensity in the following forms:

$$
\mathbf{U}_{\mathbf{o p t}}^{(1 \alpha)}(\varphi)=\arg \min _{\bar{U}^{(1 \alpha)}(\varphi)} J ; \mathbf{U}_{\mathbf{o p t}}^{(2 \alpha)}(\varphi)=\arg \min _{\bar{U}^{(2 \alpha)}(\varphi)} \varphi_{k},
$$

where $\overline{\mathbf{U}}^{(\beta \alpha)}(\varphi)$ - vector-function of autonomous control by heat sources for each $\alpha$-th verge:

$$
\overline{\mathbf{U}}^{(\beta \alpha)}(\varphi)=\left[U_{x}^{(\beta 3)}(\varphi), U_{x}^{(\beta 4)}(\varphi), U_{y}^{(\beta 5)}(\varphi), U_{y}^{(\beta 6)}(\varphi), U_{z}^{(\beta 1)}(\varphi), U_{z}^{(\beta 2)}(\varphi)\right]
$$

in maximum accuracy problem $(\beta=1)$ and for task performance $(\beta=2)$, then using the method of moments $[2,4]$ both problems can be parameterized and reduced to the problem of searching for a finite number of parameters $\Delta_{\delta}^{(i)}, \delta=1,2, . ., i ; i=1,2, . . J$; here $\Delta_{\delta}^{(i)}$ - duration of $\delta$-th interval from the total set of $i$ intervals, where each component of the vector $\bar{U}^{(\beta \alpha)}(\varphi)$ alternately takes its possible values at the level of restrictions (7).

Duration $\Delta_{\delta}^{(i)}$ and the required number of intervals, $i$, can be determined using alternance [4] method that allows to write the following system of transcendental equations:

$$
\left.\Theta\left(\rho_{k}, \varphi_{k}\right)\right|_{\rho_{k} \in \bar{D}_{k}}= \pm \varepsilon^{(i)},\left.\operatorname{grad} \Theta\left(\rho_{k}, \varphi_{k}\right)\right|_{\rho_{k} \in \bar{D}_{k}}=0
$$

where

$\Theta=\int_{0}^{\varphi_{k}} \int_{0}^{l_{x}} \int_{0}^{l_{y}} \int_{0}^{l_{z}} G\left(\varphi-\varphi^{\prime}, l_{x}, l_{y}, l_{z}, l_{x}^{\prime}, l_{y}^{\prime}, l_{z}^{\prime}\right) \cdot \omega\left(l_{x}^{\prime}, l_{y}^{\prime}, l_{z}^{\prime}, U_{o p t}^{(\beta \alpha)}\left(\Delta_{\delta}^{(i)}\right), \varphi\right) d \varphi^{\prime} d l_{x}^{\prime} d l_{y}^{\prime} d l_{z}^{\prime}$

- the resulting solution of boundary problem (1)-(4) in the final time instance $\varphi_{k}$, $\rho_{k}\left(l_{x}, l_{y}, l_{z}\right) \in \rho\left(l_{x}, l_{y}, l_{z}, \varphi_{k}\right) \in \bar{D}_{k}$ - countable set of limit points, where the relations (11) are valid. These limit points belong to the closed set $\bar{D}_{k} \subseteq \bar{\Omega}_{p} \subset E^{3}$ of definition domain $\bar{\Omega}_{p}$ for function $\Theta\left(\rho_{k}, \varphi_{k}\right), G\left(\varphi-\varphi^{\prime}, l_{x}, l_{y}, l_{z}, l_{x}^{\prime}, l_{y}^{\prime}, l_{z}^{\prime}\right)$ is a Green's function for the boundary problem (1)-(4), $\varepsilon^{(i)}=\max \left|\Theta\left(\rho, \Delta_{\delta}^{(i)}, \varphi_{k}\right)\right|$ is the maximum deviation of $T\left(x, y, z, \tau_{k}\right)$ from the desired value $T_{\text {dest }}$ that can be reached in the class of $i$-intervals control functions. It should be noted that there is a limit $\varepsilon_{\text {inf }}$ of achievable deviations and thus the limiting number $J$ of intervals $\Delta_{\delta}^{(i)}$, $\delta=1,2, . ., i ; \quad i=1,2, . . J$ of optimal control; and the following condition holds true: $\varepsilon^{(1)}>\varepsilon^{(2)}>\varepsilon^{(3)}>. .>\varepsilon^{(J)}=\varepsilon_{\text {inf }}$. In the case of autonomous control of each $j$-th, $j=\overline{1, M}$ of $M=\sum_{\alpha=1}^{6} M_{\alpha}$ controlled heat sources $U_{j}^{(\alpha)}(\varphi), \alpha=\overline{1,6}$, arranged according to (1) - (4) on the respective areas $V_{x j}^{(\alpha)}\left(l_{y}, l_{z}\right), \alpha=3,4 ; V_{y j}^{(\alpha)}\left(l_{x}, l_{z}\right), \alpha=5,6 ; V_{z j}^{(\alpha)}\left(l_{x}, l_{y}\right), \alpha=1,2$; the 
expression (8) takes the form: $q_{x}^{(\alpha)}\left(\varphi, l_{y}, l_{z}\right)=\sum_{\alpha=1}^{6} U_{j}^{(\alpha)}(\varphi) V_{x j}^{(\alpha)}\left(l_{y}, l_{z}\right), \alpha=3,4 ; \alpha=5,6$ $q_{y}^{(\alpha)}\left(\varphi, l_{x}, l_{z}\right)=\sum_{\alpha=1}^{6} U_{j}^{(\alpha)}(\varphi) V_{y j}^{(\alpha)}\left(l_{x}, l_{z}\right) ; q_{z}^{(\alpha)}\left(\varphi, l_{x}, l_{y}\right)=\sum_{\alpha=1}^{6} U_{j}^{(\alpha)}(\varphi) V_{z j}^{(\alpha)}\left(l_{x}, l_{y}\right)$, $\alpha=1,2$; In this case a vector control function (10) for each of the formulated problems $(\beta=1,2)$ comprises $M$ components $\mathbf{U}^{(\beta)}(\varphi)=\mathbf{U}_{\mathbf{j}}^{(\beta \boldsymbol{\alpha})}(\varphi), j=\overline{1, M}$, and the alternance method procedure holds the defining relations (11) valid.

\section{Conclusion}

The proposed procedure for solving problem of optimal control of the temperature distribution of the construction can be numerically implemented using different software, for example ANSYS, that allows to obtain the results suitable for the approximate realization of autonomous system using onboard computers and significantly reduces thermal-component measurement error.

\section{References}

1. M.Y. Livshits, M.Y. Derevjanov, S.A. Kopytin, Distributed temperature control of the structural elements of autonomous objects (XIV Minsk International Forum on Heat and Mass Transfer: abstracts and reports, 2012)

2. A.G. Butkovskij, Strukturnaya teoriya raspredelennyx sistem (M. Nauka, 1977)

3. A.G. Shashkov, Sistemno-strukturny'j analiz processa teploobmena i ego primenenie (M. E'nergoatomizdat, 1983)

4. E'.Ya. Rapoport, Optimal'noe upravlenie sistemami s raspredelenny'mi parametrami (M. Vy'sshaya shkola, 2009)

5. V.P Roizman, Mechanics in electronics (Hmel'nickij : HNU, 2015) 